\title{
Molecular Insights on Successful Reconstitution of Freeze-Dried Nanofibrillated Cellulose Hydrogel
}

\author{
Koivunotko, Elle
}

2021-09-20

Koivunotko , E , Merivaara , A, Niemela , A, Valkonen , S, Manninen , K, Makinen , H , Viljanen, M , Svedstrom , K, Diaz , A , Holler , M , Zini , J , Paasonen , L , Korhonen , O , Huotari , S , Koivuniemi , A \& Yliperttula , M 2021, ' Molecular Insights on Successful Reconstitution of Freeze-Dried Nanofibrillated Cellulose Hydrogel ' , ACS Applied Bio Materials, vol. 4 , no. 9 , pp. 7157-7167 . https://doi.org/10.1021/acsabm.1c00739

http://hdl.handle.net/10138/335295

https://doi.org/10.1021/acsabm.1c00739

cc_by

publishedVersion

Downloaded from Helda, University of Helsinki institutional repository.

This is an electronic reprint of the original article.

This reprint may differ from the original in pagination and typographic detail.

Please cite the original version. 


\title{
Molecular Insights on Successful Reconstitution of Freeze-Dried Nanofibrillated Cellulose Hydrogel
}

\author{
Elle Koivunotko, Arto Merivaara, Akseli Niemelä, Sami Valkonen, Kalle Manninen, Henrik Mäkinen, \\ Mira Viljanen, Kirsi Svedström, Ana Diaz, Mirko Holler, Jacopo Zini, Lauri Paasonen, Ossi Korhonen, \\ Simo Huotari, Artturi Koivuniemi, and Marjo Yliperttula*
}

Cite This: ACS Appl. Bio Mater. 2021, 4, 7157-7167

Read Online

ABSTRACT: The diversity and safety of nanofibrillated cellulose (NFC) hydrogels have gained a vast amount of interest at the pharmaceutical site in recent years. Moreover, this biomaterial has a high potential to be utilized as a protective matrix during the freezedrying of heat-sensitive pharmaceuticals and biologics to increase their properties for long-term storing at room temperature and transportation. Since freeze-drying and subsequent reconstitution have not been optimized for this biomaterial, we must find a wider understanding of the process itself as well as the molecular level interactions between the NFC hydrogel and the most suitable lyoprotectants. Herein we optimized the reconstitution of the freeze-dried NFC hydrogel by considering critical quality attributes required to ensure the success of the process and gained insights of the obtained experimental data by simulating the effects of the used lyoprotectants on water and NFC. We discovered the correlation between the measured characteristics and molecular dynamics simulations and obtained successful freezedrying and subsequent reconstitution of NFC hydrogel with the presence of $300 \mathrm{mM}$ of sucrose. These findings demonstrated the possibility of using the simulations together with the experimental measurements to obtain a more comprehensive way to design a successful freeze-drying process, which could be utilized in future pharmaceutical applications.

KEYWORDS: nanofibrillated cellulose hydrogel, trehalose, sucrose, freeze-drying, rheology, residual water content, molecular dynamics simulations, ptychographic X-ray computed tomography

\section{INTRODUCTION}

Nanofibrillated cellulose (NFC) hydrogel is a natural biomaterial, whose biocompatibility, noninvasive properties, and hydrophilicity have aroused interest in many research sites, such as in $3 \mathrm{D}$ cell culturing and biomedical applications. ${ }^{1,2}$ Furthermore, the optimization of the drying of NFC hydrogel into a highly porous aerogel has been a subject of research ${ }^{3,4}$ to be utilized in various chemical applications as well as in pharmaceutical ones. However, the tendency of the hydrophilic fibrils of the nanocellulose to form irreversible hydrogen bonds between each other and aggregate during the drying process have created obstacles in the drying trials of $\mathrm{NFC}^{5}$ and prevents the reconstitution back to hydrogel. Although previously NFC hydrogel has been successfully dried into a porous aerogel form, its applicability to be later reconstituted back to hydrogel with preserved material-specific properties has not been comprehensively evaluated. This stands as a motivation to further study freeze-drying and successful reconstitution of this material. To better achieve the optimal freeze-drying protocol and reconstitution for native NFC hydrogel a detailed understanding of the interactions between NFC, water, and utilized lyoprotec- tants at the atomic scale would provide the means to optimize this method for different pharmaceutical approaches.

In general, freeze-drying is considered as one of the major formulation techniques in the biopharmaceutical industry to improve the shelf life of thermosensitive products, such as vaccines. ${ }^{6}$ In addition, freeze-drying has been studied for longterm preservation and more feasible transportation of biomaterials and even living substances, such as cells. ${ }^{7}$ The optimized freeze-drying cycle consists of three main steps: (1) freezing where the water of the sample is frozen until the fully freeze-concentrated form is reached, (2) primary drying where the frozen parts are removed via sublimation under a vacuum and below a glass transition temperature $\left(T_{\mathrm{g}}{ }^{\prime}\right)$, and (3)

Received: June 28, 2021

Accepted: August 24, 2021

Published: September 1, 2021

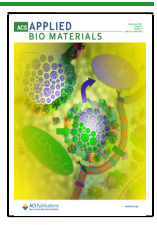


secondary drying where the unfrozen water of the sample is desorbed at elevated temperatures. ${ }^{6}$ The first things to determine after the freeze-drying process and possible longterm storage are cake appearance and reconstitution, which are one of the quality attributes of the freeze-dried (FD) samples to ensure the success of the process. ${ }^{8}$

Since the sample is exposed to a taxing freeze-drying process, lyoprotectants are often required to preserve the characteristics of the sample and to ensure the successful reconstitution of the material later. ${ }^{9-11}$ Lyoprotectants act as thermodynamic stabilizers and/or kinetic stabilizers. Thermodynamic stabilizers have an ability to replace the hydrogen bonds of water, known as the water replacement hypothesis, whereas kinetic stabilizers form a highly viscous, degradation reducing matrix around the sample, considered as the glass dynamic hypothesis. Sugars, such as trehalose ( $\alpha$-D-glucopyranosyl- $\alpha$-D-glucopyranoside) and sucrose ( $\beta$-D-fructofuranosyl- $\alpha$-D-glucopyranoside), are commonly used as lyoprotectants as they have high glass transition temperatures and hydrogen bonding abilities. From here on these disaccharides are referred to as biomolecules. In addition to sugars, other small molecules with lyoprotective properties have been utilized in freeze-drying. These for instance include alcohols, amino acids, and organic solvents. Optimization of the quantity of the suitable lyoprotectants, taking into account sample-specific quality attributes, such as isotonicity, rheological features, desired residual water content, and morphology, is often based on the trial-and-error approach, which requires a vast amount of experimental data. ${ }^{12}$ Furthermore, according to the opinion of Patel et al. ${ }^{8}$ the ambiguity in understanding the specific quality attributes of the FD sample challenges the optimization of the freeze-drying process, which is why they should be more properly defined.

In our previous study, we showed that anionic nanofibrillated cellulose (ANFC) hydrogel could be FD and reconstituted after with preserved rheological properties, which reflected a recovered drug release profile. ${ }^{13}$ Although potential results were obtained, careful optimization of the freeze-drying process was lacking which led to the samples containing a too high water content according to the criteria stated by May et al.: ${ }^{14}$ "For most products levels of residual moisture should be low, usually from less than $1.0 \%$ to $3.0 \%$, so that the viability, immunologic potency and therefore the stability of the product is not compromised over time". Besides, residual water content is to be one of the major critical quality attributes of the FD product as noted by Kauppinen et al. ${ }^{15}$ Residual water has a major impact on, for instance, the storage stability of the FD sample as well as on the reconstitution and preservation properties, which is why it should be accurately quantified and optimized. Furthermore, especially when designing freeze-drying protocol suitable for cells and biologics the critical quality attributes are not limited to the outcome but also the optimization of the environment surrounding them during the process. The importance of understanding these aspects was noticed in another study of ours where NFC hydrogel was utilized in the freeze-drying of hepatocellular carcinoma 3D cell spheroids. ${ }^{16}$ Due to the lack of full awareness of the effects of the interactions between the cells, their extracellular matrix and lyoprotectants during freezedrying, as well as the absence of the comprehensive quality attributes for the FD sample, there were challenges in preserving the functionality of these 3D cell spheroids.

Due to the complexity of freeze-drying, there are few studies that explore the process starting from the microscopic aspect by simulating the molecular level interactions between the active component, water, and used lyoprotectants utilizing different in silico models. ${ }^{9,12,17}$ Understanding the behavior of the used lyoprotectants, such as the conformation and hydrogen bonding ability, could open new opportunities in the designing of formulations for freeze-drying and even in optimizing the desired water content for the biologics. Molecular dynamics (MD) simulations have been suggested as a new approach to explain and predict outcomes of the freeze-drying processes, ${ }^{12}$ which could minimize the time and costs compared with the optimization based only on the obtained experimental data.

To summarize, multiple aspects in the optimization of the freeze-drying process have been studied by utilizing different quality attributes of the FD sample and by simulating the molecular level interactions between the lyoprotectants and the sample formulation to be FD. However, as far as we are aware, there have not been any studies with biomaterial, biologics, or biomolecules, in which all these aspects have been concerned, starting from the standard characterization, such as cake appearance, residual water content, or via rheological and morphological analysis into more complex atomistic MD simulations of the studied system.

In this study, we aimed to optimize the freeze-drying and reconstitution of native NFC hydrogel with biomolecules by understanding the preservation of the physicochemical characteristics and rheological properties as well as the morphology utilizing the obtained information from atomistic molecular dynamics simulations. This provides valuable information on how the understanding of the behavior of biomaterial, biomolecules, and water should be combined to the designing process of freeze-drying. We hypothesized that the MD simulations could be utilized to understand the successful freeze-drying and subsequent reconstitution of native NFC hydrogel with the optimized quantity of biomolecules.

\section{EXPERIMENTAL SECTION}

2.1. NFC Hydrogel Preparation. Native nanofibrillated cellulose (NFC) hydrogel (GrowDex®) was provided by UPM, Biomedicals, Finland, with a fiber content of $1.5 \%(\mathrm{~m} / \mathrm{v})$. The selection of the final biomolecules was based on the optimization process presented in the Supporting Information (SI), Figure S1. The final biomolecules used in this study, D-(+)-trehalose dihydrate and sucrose, were purchased from Sigma-Aldrich, U.S.A. The studied NFC hydrogel formulations were prepared by mixing different concentrations of biomolecules with ultrapure water, which were then combined with NFC hydrogel to the total fiber content of $0.8 \%(\mathrm{~m} / \mathrm{v})$. The total concentration of the biomolecules in NFC hydrogel formulations was between 100 and 1000 $\mathrm{mM}$. NFC hydrogel formulations were then homogenized by mixing them with the vortex, and samples with higher concentrations than 600 $\mathrm{mM}$ of the biomolecules were mixed with the syringe technique based on the protocol from Paukkonen et al. ${ }^{13}$ NFC hydrogel with a fiber content of $0.8 \%(\mathrm{~m} / \mathrm{v})$ without any biomolecules was used as a control.

2.2. Freeze-Drying Protocol. To optimize freeze-drying temperatures, the glass transition temperatures of maximally freezeconcentrated samples $\left(T_{\mathrm{g}}{ }^{\prime}\right)$ of all the NFC hydrogel formulations and the control were measured with differential scanning calorimetry (DSC; Discovery 2500, TA Instruments, Germany). Samples were pipetted to hermetically sealed T-Zero pans $(40 \mu \mathrm{L})$. Measurements were carried out by first decreasing the temperature from +40 to -80 ${ }^{\circ} \mathrm{C}$ and then heating $10{ }^{\circ} \mathrm{C} / \mathrm{min}$ to $+20^{\circ} \mathrm{C}$ with a cell purge gas flow (N2) $50 \mathrm{~mL} / \mathrm{min}$. Temperature and heat flow was calibrated with indium. Glass transition temperature measurements were done as triplicates, and results were analyzed with TRIOS software (TA Instruments, Germany).

Freeze-drying was performed with a laboratory-scale freeze-dryer, Lyostar II (SP Scientific Inc., U.S.A.). The freezing step was performed by decreasing temperature $1{ }^{\circ} \mathrm{C} / \mathrm{min}$ to $-47^{\circ} \mathrm{C}$ for $2 \mathrm{~h}$, after which the 
A

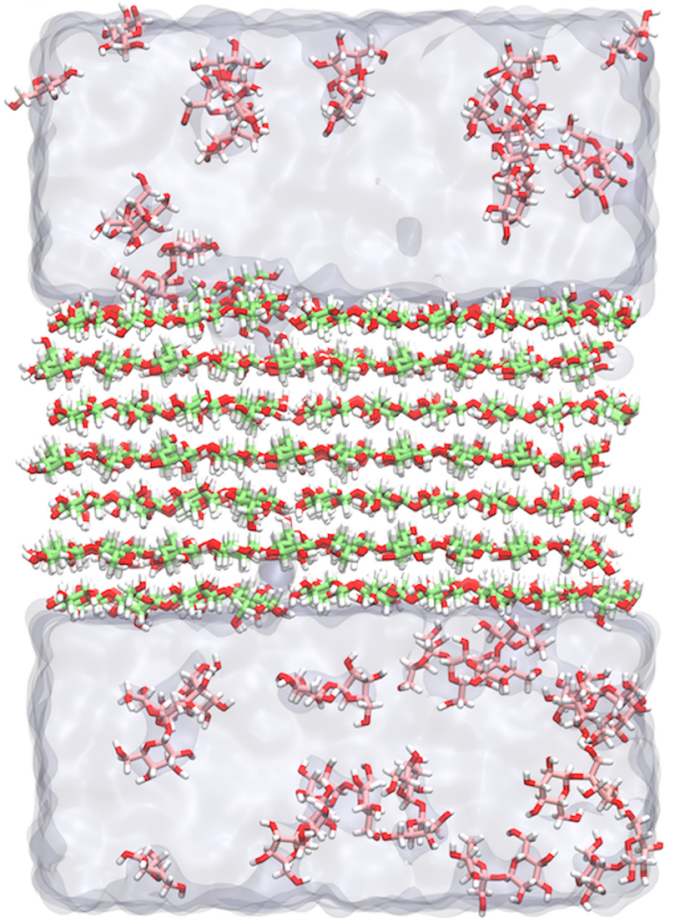

B

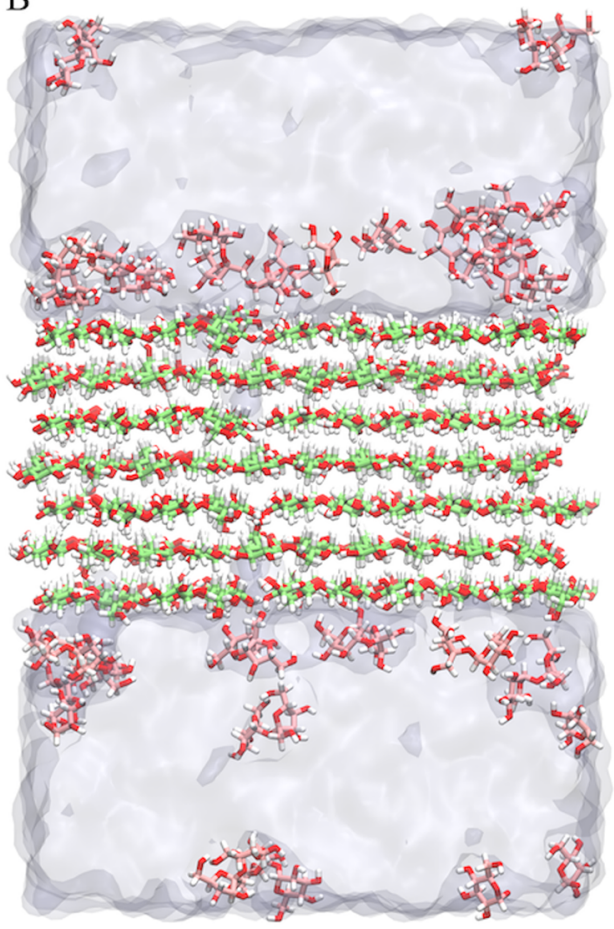

Figure 1. (A) Snapshot of the hydrophobic plane MD system at a starting point and (B) at equilibrium after the addition of biomolecules (sucrose in this example). Nonwater molecules are rendered as sticks and colored according to atom type. Carbon atoms of cellulose are green and pink for sucrose, oxygen atoms are red, and hydrogen atoms white. Water is rendered as a transparent surface.

pressure was decreased to $50 \mathrm{~m}$ Torr and temperature was increased 1 ${ }^{\circ} \mathrm{C} / \mathrm{min}$ to $-42{ }^{\circ} \mathrm{C}$. Primary drying was determined with a comparative pressure measurement using a capacitance manometer (absolute pressure, CM) and the Pirani gauche (relative vacuum). After the Pirani value had decreased under the capacitance value, freeze-drying was then continued to the secondary drying where the temperature was gradually increased $1{ }^{\circ} \mathrm{C} / \mathrm{min}$ from -42 to $+20^{\circ} \mathrm{C}$. The sample volume was 0.2 or $1.75 \mathrm{~mL}$ depending on the intended experiment. NFC hydrogel formulations and control were FD in Schott Toplyo injection vials (Adelphi) with a volume of 2 or $6 \mathrm{~mL}$ depending on the sample size. Vials were closed with rubber Daikyo D Sigma freeze-drying stoppers (Adelphi) at dry nitrogen atmosphere. After the freeze-drying all-aluminum crimp seals (West Pharmaceutical Services) were placed on the vials. FD samples were stored at $+4{ }^{\circ} \mathrm{C}$ before further experiments.

2.3. Cake Appearance and Reconstitution. After freeze-drying, the elegance of the formed cakes was evaluated, and the FD NFC hydrogel formulations and the FD control were reconstituted with ultrapure water until the starting volume immediately after opening the cap of the vial. The rate of the reconstitution of the dry samples into hydrogel and preservation of the uniformity were evaluated. Air bubbles in the reconstituted hydrogels were removed with $1 \mathrm{~min}$ centrifugation (1000 rpm, $200 \mathrm{rcf})$.

2.4. Physicochemical Properties. The physicochemical properties of the reconstituted FD samples were studied by measuring the osmotic pressure and $\mathrm{pH}$, which were compared with the results before the freeze-drying. Osmotic pressure was measured with a manual freezing point osmometer (Osmomat 3000, Gonotech, Germany). The osmometer was calibrated for $0 \mathrm{mOsmol} / \mathrm{kg}$ with ultrapure water and $100-850 \mathrm{mOsmol} / \mathrm{kg}$ with calibration standards ( $\mathrm{NaCl}$, Gonotech). $\mathrm{pH}$ was measured with $\mathrm{pH}$ paper (Macherey-Nagel, Germany).

2.5. Rheology. Viscosity, shear loss modulus $\left(G^{\prime \prime}\right)$, and shear storage modulus $\left(G^{\prime}\right)$ measurements were performed with HAAKE Viscotester iQ Rheometer (Thermo Scientific, Karlsruhe, Germany). The instrument included the Peltier system for temperature control, and all of the measurements were done at $24{ }^{\circ} \mathrm{C}$. Data from the rheological measurements were analyzed with HAAKE RheoWin 4.0 software (Thermo Fischer Scientific, U.S.A.) and with OriginPro (OriginLab, U.S.A.). The samples were left to equilibrate to room temperature before the measurements. Measurements were taken after the reconstitution of the FD samples and compared with the ones taken before the freeze-drying.

For the shear viscosity measurements, plate-on-plate geometry (1 $\mathrm{mm}$ gap) with parallel $35 \mathrm{~mm}$ diameter steel was used. The shear rate was increased from 0.1 to $1000 \mathrm{~s}^{-1}$, and the results were observed with 16 time points. Oscillatory frequency sweep analysis was performed with double gap geometry ( $4 \mathrm{~mm}$ gap) with parallel $25 \mathrm{~mm}$ diameter steel. Before the measurement, constant amplitude sweeps were performed for the determination of the linear viscoelastic region. Constant angular frequency $\omega=1 \mathrm{~Hz}$ with oscillatory stress between 1 $\times 10^{-4}-500 \mathrm{~Pa}$ was used. Based on the linear viscoelastic region, the chosen oscillatory stress was $\tau=1.5 \mathrm{~Pa}$ and the angular frequency range was $0.6-18.5 \mathrm{rads}^{-1}$. The results from the moduli were observed with 24 time points.

2.6. Residual Water Content. Residual water contents of the FD NFC hydrogel formulations and the FD control were measured with an automatic coulometric Karl Fischer titrator (Metrohm, 899 Coulometer, Switzerland). Hydranal (Coulomat AG, Fluca) was used as a solvent in the measurements. FD samples were mixed with $1 \mathrm{~mL}$ of methanol and drawn into a syringe with a volume of $0.8 \mathrm{~mL}$, which were injected into a bath flask of the Karl Fischer titrator. Results were given in micrograms of water and the water content of pure methanol was taken into account as a blank in the calculations. Mass percent and mole fraction of the residual water content in the FD samples were calculated manually. The molecular weight of NFC was considered as the molecular weight of the glucose monomer in the calculations and the mole fraction of water was theoretically calculated to be over $99 \%$ in all of the NFC hydrogel formulations and the control before freeze-drying.

2.7. Scanning Electron Microscopy (SEM). The morphology of the FD NFC hydrogel formulations and the FD control was studied with a scanning electron microscope Quanta FEG250 (SEM, FEI Company, U.S.A.). The sample preparation was done manually by cutting the FD sample with tweezers for detection of the inner structure and surface analysis of the sample. Samples were placed on two-sided 
carbon tape with silver paint. Before the imagining, samples were sputtered with platinum for $25 \mathrm{~s}$ with an Agar sputter instrument (Agar Scientific Ltd., U.K.). Samples were imaged in a high vacuum with 6-7 $\mathrm{kV}$ and $2-4$ spot size.

2.8. Ptychographic X-ray Computed Tomography (PXCT). FD NFC hydrogel formulation including $300 \mathrm{mM}$ of sucrose and FD control were studied with ptychographic X-ray computed tomography (PXCT),${ }^{18}$ conducted at the coherent small-angle X-ray scattering beamline of the Swiss Light Sources (SLS), PSI, Switzerland, using the OMNY instrument. ${ }^{19}$ The measurements were conducted at a temperature of about $90 \mathrm{~K}$ using a photon energy of $6.2 \mathrm{keV}$. A Fresnel zone plate with a diameter of $220 \mu \mathrm{m}$, an outermost zone width of 60 $\mathrm{nm}$, and a focal distance of $66 \mathrm{~mm}$ was used to define a coherent, structured illumination onto the FD sample. ${ }^{20}$ Near-field ptychography measurements were performed with the sample placed at $9.5 \mathrm{~mm}$ downstream of the focus, where the illumination on the sample had a size of about $32 \mu \mathrm{m}$. Ptychographic projections were recorded by scanning the FD sample in the beam following a Fermat spiral pattern with an average step size of $4 \mu \mathrm{m}$. At each scanning position, we acquired X-ray diffraction patterns with an in-vacuum Eiger $1.5 \mathrm{M}$ detector (detector group, PSI), placed $7.204 \mathrm{~m}$ downstream of the FD sample.

The data recorded from ptychography scans were processed to obtain 2D projections of the sample. For this purpose, near-field ptychographic reconstructions were performed using the ptychoShelves package (Coherent X-ray Scattering Group, PSI) ${ }^{21}$ with a combination of the difference map algorithm followed by a maximum likelihood optimization algorithm. ${ }^{22} \mathrm{X}$-ray ptychography is often performed in the far-field, for which ptychographic algorithms were originally written. Near-field ptychography uses similar algorithms, except for the propagation of the wave-field to the detector, as described in the previous work. ${ }^{23,24}$ The pixel size of the images was $99.0 \mathrm{~nm}$, determined by the geometrical magnification on the detector.

Ptychographic scans were repeated at different incidence angles of the FD sample with respect to the X-ray beam in regular angular steps ranging from $0^{\circ}$ to $180^{\circ}$. The projections were aligned, and $3 \mathrm{D}$ tomograms were computed using a modified filtered back-projection reconstruction with a GPU-based Matlab routine. ${ }^{25}$ The $3 \mathrm{D}$ resolution of the resulting tomograms was estimated by Fourier shell correlation (FSC). Tomographic reconstruction from phase projections provides a quantitative 3D map of the electron density within the specimen. ${ }^{26}$ The $3 \mathrm{D}$ rendered volumes were visualized using the VolumeViewer of Fiji. ${ }^{27}$ The coding of the parallel samples and the parameters of the scans of the presented FD samples are shown in more detail in the SI, Table S1.

2.9. Atomistic Molecular Dynamics Simulations. To investigate the interactions between trehalose or sucrose and NFC, atomistic molecular dynamics (MD) simulations were carried out. Three planelike simulation systems were constructed: cellulose in a crystalline morphology to determine the binding free energies, one with a hydrophobic surface and one with a hydrophilic surface, and cellulose in an amorphous morphology to determine the effects of sugars on water penetration. The systems were built with the help of the cellulosebuilder toolkit and the protocol of Bregado et al. ${ }^{28}$ Construction of the simulation systems and MD analyses are described in more detail in the SI. Figure 1 shows the MD system at the beginning of the simulation and at equilibrium after the addition of the biomolecules.

2.9.1. Simulation Force Field and Parameters. The CHARMM 36 force field was used for the cellulose, trehalose, and sucrose parameters, obtained from CHARMM-GUI for GROMACS. ${ }^{29}$ TIP3P model was used for water. ${ }^{30}$ For final production runs a v-rescale thermostat at 300 $\mathrm{K}$ was used with a coupling constant of $1.0 \mathrm{ps}$ and a semi-isotropic Parinello-Rahman barostat at 1.0 bar with a 5.0 ps coupling constant. $^{31,32}$ Lennard-Jones interaction cut-offs were set to $1.0 \mathrm{~nm}$ and electrostatics were described with a Particle-Mesh Ewald summation scheme with a $1.2 \mathrm{~nm}$ cutoff. $^{33}$ The final production simulations were run with the GROMACS simulation package (2020.1) with a time step of $2 \mathrm{fs}$ with the leapfrog algorithm. ${ }^{34}$ Minimizations utilized the steepest descent algorithm.

2.9.2. Solvation of Systems and the Addition of Biomolecules. After the equilibration of the cellulose planes, the periodic boundary condition box's z-dimension was tripled in size, and the cellulose plane recentered on it. Water solvation was carried out with gmx solvate, which resulted in 2851 water molecules for crystalline systems and 3123 for the amorphous system. Solvated NFC systems were simulated without biomolecules to equilibrate the number of hydrogen bonds between the water and cellulose. Ultimately, solvated NFC simulations were continued until $1 \mu \mathrm{s}$. The amorphous and the hydrophilic crystalline systems were in equilibrium after $\sim 500 \mathrm{~ns}$ and the hydrophobic crystalline system after $\sim 200$ ns. Consequently, biomolecules were randomly placed into the water phase. Therefore, two new systems were generated from each NFC system with $300 \mathrm{mM}$ of trehalose or $300 \mathrm{mM}$ of sucrose. This resulted in amorphous systems having 30 biomolecules and hydrophobic and hydrophilic crystalline systems 24 . Furthermore, to gauge the repeatability of the simulations, we performed duplicates of the amorphous and hydrophobic crystalline systems containing biomolecules. After minimization, all systems were run until $1 \mu \mathrm{s}$. Again, the number of hydrogen bonds between cellulose and water was used as a metric for equilibration. All systems apart from one equilibrated readily so the starting point for analyses was set to 40 ns. The system with a hydrophobic plane and $300 \mathrm{mM}$ of trehalose equilibrated after $\sim 600 \mathrm{~ns}$. Thus, this system was simulated until $1.1 \mu \mathrm{s}$ to get $500 \mathrm{~ns}$ long equilibrium trajectories for analysis of each system. The equilibrium plots are presented in the SI, Figure S3.

\section{RESULTS}

According to the NFC hydrogel optimization criteria described in the SI, Figure S1, the presented data concentrates on the last two most suitable NFC hydrogel formulations, including 300 $\mathrm{mM}$ of trehalose and $300 \mathrm{mM}$ of sucrose, which had the highest $T_{\mathrm{g}}{ }^{\prime}$ values (SI Table S2). The data of the residual water contents included also the results of the other NFC hydrogel formulations to evaluate more clearly the mole fraction differences of water.

3.1. External Appearance Showed Similarities between the FD NFC Cakes. The appearances of FD NFC cakes were evaluated after freeze-drying to confirm the success of the process. The elegant white solid cakes were obtained with the FD NFC hydrogel formulations including $300 \mathrm{mM}$ of trehalose or sucrose. Surprisingly, the FD control had a similar cake appearance compared with these two FD NFC hydrogel formulations, and only a slight difference in the cake structure could be observed (Figure 2). However, after the reconstitution, the differences between the samples including $300 \mathrm{mM}$ of the biomolecules and the control could be observed. With the presence of $300 \mathrm{mM}$ of the biomolecules, the color of the cake changed to transparent immediately after reconstitution, and hydrogel was formed after 2 min of gentle, manual stirring, whereas the control formed aggregates, which were impossible to homogenize. To remove formed air bubbles inside of the successfully reconstituted FD NFC hydrogel formulations, the samples were centrifuged, after which they corresponded precisely to the ones before the freeze-drying.

3.2. Rheological Properties of the NFC Hydrogel Were Preserved with the Presence of Sucrose. To ensure the preserved structural properties and homogeneity of the FD and subsequently reconstituted samples, rheological measurements were implemented, which are sensitive to the changes of these features. The results were compared with the ones before the freeze-drying process. Before the freeze-drying, viscosity and viscoelasticity did not differ whether the biomolecules were added or not into the NFC hydrogel (Figure 3A,B). However, after the reconstitution of the FD samples, rheological properties showed significant differences between the control and the samples including $300 \mathrm{mM}$ of trehalose or sucrose. Unlike in the control, linearly decreasing shear viscosity as well as elastic properties, where $G^{\prime}$ was above $G^{\prime \prime}$, were preserved with the 

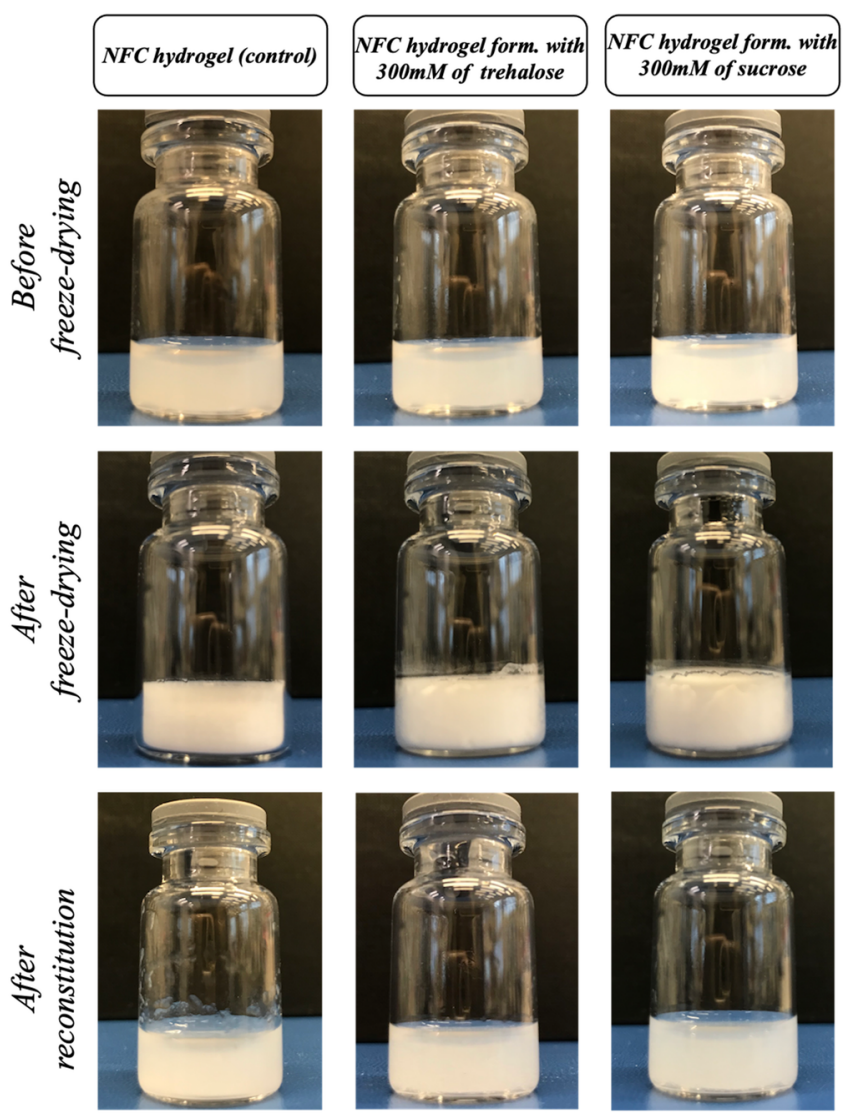

Figure 2. NFC hydrogel appearance before freeze-drying, after freezedrying, and right after reconstitution before the air bubble removing. Samples contained $1.75 \mathrm{~mL}$ of NFC hydrogel (control, left), NFC hydrogel formulation with $300 \mathrm{mM}$ of trehalose (middle), and with 300 $\mathrm{mM}$ of sucrose (right). Differences between the control and the NFC hydrogel formulations with sugars could be observed after the reconstitution of the FD sample: aggregation of the control could be observed from the edges of the vial while two other samples formed homogeneous hydrogels equal to the one before freeze-drying. The uneven surface of the FD cakes is caused by the movement of the hydrogel form before they were dried. Abbreviations: NFC = nanofibrillated cellulose, form. $=$ formulation, $\mathrm{mM}=$ millimolar.

presence of these biomolecules. A slight decrease in both the viscosity and the $G^{\prime \prime}$ in viscoelastic measurements could be observed with the reconstituted FD NFC hydrogel formulation including trehalose whereas the one with sucrose showed equal results in the rheological properties before and after the freezedrying and reconstitution.

In addition to the slight differences between the biomolecules in preserving the rheological features of the NFC hydrogel, the calculated residual water contents showed variation between the FD samples including trehalose or sucrose. All the FD NFC hydrogel formulations showed the mass percent of the residual water content to be under $1.6 \%$ (Figure 3C). To illustrate in more detail the proportion of water in the dried form, mole fractions of water were calculated. This revealed differences between the FD samples as observed from Figure 3C. When the concentration of trehalose was increased, the mole fraction of water was relatively stable with a range between $8.27 \%$ and $11.4 \%$. The mole fraction of water in the FD NFC hydrogel formulation with sucrose was increased with increasing sugar concentration from $4.95 \%$ to $18.6 \%$. This was a stronger increase compared with the ones including trehalose. In the concen- tration range of 100 and $300 \mathrm{mM}$ the proportionality between the increasing sucrose concentration and water amount could be observed. A slight plateau in the mole fraction of water is seen in the concentration range of $300 \mathrm{mM}-700 \mathrm{mM}$ of FD NFC hydrogel formulation with sucrose.

\subsection{Organized and Homogeneous Microstructure of} the FD Cake with the Presence of Sucrose. Morphology was evaluated from the FD NFC hydrogel formulation including $300 \mathrm{mM}$ of trehalose or sucrose and the FD control utilizing scanning electron microscopy (SEM). Differences in the structure between the FD samples can be observed from the SEM micrographs in Figure 4A. The FD control (above) showed an accumulated, lamellar structure, which differed significantly from the FD NFC hydrogel formulation including sucrose (below) or trehalose (SI, Figure S5). With the presence of sucrose, a more organized structure was observed with symmetrical pores compared with the FD control, which was also observed from the SEM micrographs taken from the FD NFC hydrogel formulation including trehalose.

Differences between the morphology of the most promising FD NFC hydrogel formulation with $300 \mathrm{mM}$ of sucrose and the FD control were assessed in more detail by determining the 3D structure and electron densities with PXCT. 3D structure of the FD control presented in Figure 4B (above, control 1) showed a highly unorganized surface with distinguishable microfibre structure that could also be observed from the cross-section images of this tomogram where the width of the structures varied with the mean being $380 \mathrm{~nm}$ (Figure 4C, above). Interestingly, a high variation between the measurements of the parallel samples of the FD control was detected, both morphological and regarding the observed electron densities. As shown in the SI, Figure S6, instead of the microfibre structure, the parallel sample (control 2) showed a homogeneous bulk structure with a nonhomogenous outer layer having both lower and significantly higher electron densities. These higher densities presented as white regions were connected to the outer zones and had a mean electron density value of $0.8 \AA^{-3}$. The findings of the FD control differed significantly from the FD NFC hydrogel formulation including $300 \mathrm{mM}$ of sucrose that had evenly formed 3D structure (Figure 4B, below, FD NFC sample with sucrose 1). Furthermore, the electron density range was narrow throughout the FD sample with sucrose (Figure 4C, below), which was from 0.4 to $0.5 \AA^{-3}$. The similar features of the observed properties were presented in the parallel sample (SI, Figure S7A,B, FD NFC sample with sucrose 2). No regions of pure water were observed in any of the FD samples $\left(0.33 \AA^{-3}\right) .^{35}$

3.4. Atomistic Simulations Revealed Differing Interactions between the Molecular Components in the Different NFC Systems. To gain insight into the differences between the behavior of trehalose and sucrose in the NFC systems, $\mathrm{MD}$ simulations were performed and interactions between the biomolecules and cellulose were evaluated. Two main properties were investigated: attraction of the biomolecules on cellulose fibers and the effect of biomolecules in the penetration of water molecules into the cellulose matrix. During the simulations spontaneous peeling of singular fibers was observed after adjustment of the biomolecules.

The binding free-energy results for biomolecules showed a similar trend in all NFC systems (amorphous, crystalline hydrophilic, and crystalline hydrophobic). Namely, the results in Figure 5A show that trehalose molecules had a lower attraction $\left(\Delta G_{\text {bind }}=-1.38-(-2.79) \mathrm{kJ} / \mathrm{mol}\right)$ to the NFC surface when compared to sucrose $\left(\Delta G_{\text {bind }}=-1.63-(-3.12)\right.$ 
A

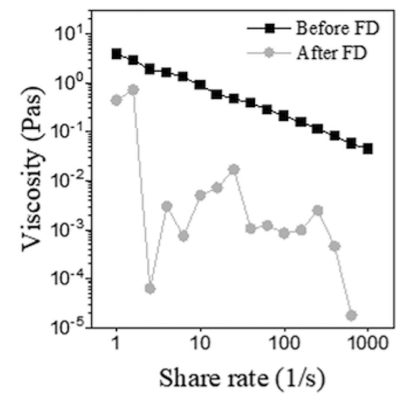

B

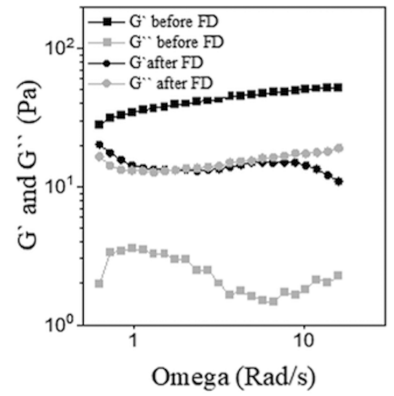

NFC hydrogel form. with $300 \mathrm{mM}$ of trehalose

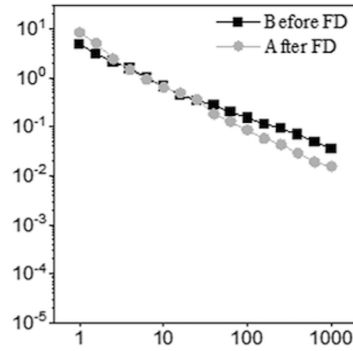

Share rate $(1 / \mathrm{s})$

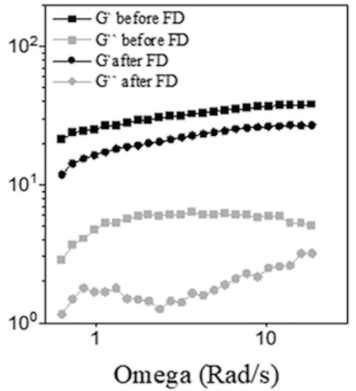

NFC hydrogel form. with $300 \mathrm{mM}$ of sucrose

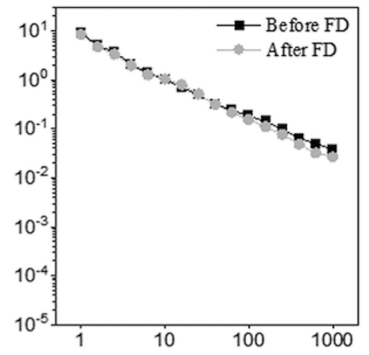

Share rate $(1 / \mathrm{s})$

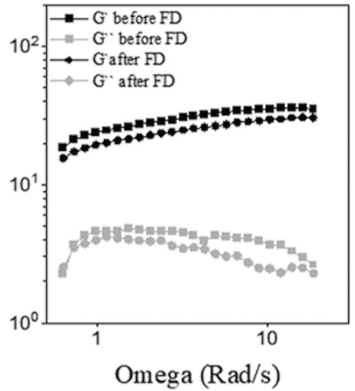

C

\begin{tabular}{|l|l|l|l|l|}
\cline { 2 - 5 } \multicolumn{1}{c|}{} & \multicolumn{2}{|l|}{ FD NFC hydrogel form. with trehalose } & \multicolumn{2}{l|}{ FD NFC hydrogel form.with sucrose } \\
\hline $\begin{array}{l}\text { Concentration of the } \\
\text { biomolecule } \\
(\text { millmolar) }\end{array}$ & $\begin{array}{l}\text { Mass percent of } \\
\text { water in \% } \\
( \pm \mathrm{SD})\end{array}$ & $\begin{array}{l}\text { Mole fraction of } \\
\text { water in \% } \\
( \pm \mathrm{SD})\end{array}$ & $\begin{array}{l}\text { Mass percent of } \\
\text { water in \% } \\
( \pm \mathrm{SD})\end{array}$ & $\begin{array}{l}\text { Mole fraction of } \\
\text { water in \% } \\
( \pm \mathrm{SD})\end{array}$ \\
\hline 100 & $0.768( \pm 0.1)$ & $9.23( \pm 0.89)$ & $0.371( \pm 0.02)$ & $4.95( \pm 0.67)$ \\
\hline 200 & $0.591( \pm 0.05)$ & $8.27( \pm 0.33)$ & $0.496( \pm 0.03)$ & $6.88( \pm 0.09)$ \\
\hline 300 & $0.734( \pm 0.1)$ & $11.4( \pm 1.5)$ & $1.00( \pm<0.01)$ & $14.9( \pm 0.04)$ \\
\hline 500 & $0.662( \pm 0.05)$ & $9.7( \pm 0.57)$ & $1.08( \pm 0.05)$ & $14.3( \pm 0.49)$ \\
\hline 700 & $1.12( \pm 0.1)$ & $11.1( \pm 1.3)$ & $1.14( \pm 0.04)$ & $14.6( \pm 0.52)$ \\
\hline 1000 & $1.15( \pm 0.2)$ & $10.1( \pm 2.0)$ & $1.56( \pm 0.1)$ & $18.6( \pm 1.1)$ \\
\hline
\end{tabular}

Figure 3. (A) Shear rate viscosity and (B) oscillatory frequency sweeps before freeze-drying and after the reconstitution of the FD cake of the NFC hydrogel (control, left), the NFC hydrogel formulation with $300 \mathrm{mM}$ of trehalose (middle) and with $300 \mathrm{mM}$ of sucrose (right). Standard deviations in the viscosity measurements from left to right were in between 0.002 and $1.8,<0.001-1.6$ and $<0.001-1.6$ before freeze-drying and $<0.001-0.5,0.02-$ 4.1 and 0.002-2.0 after freeze-drying. Standard deviations in the oscillatory frequency sweeps in average from left to right were in between 2.1 and 4.3 $\left(G^{\prime}\right)$ and $0.7-2.6\left(G^{\prime \prime}\right), 4.1-5.3\left(G^{\prime}\right)$ and $0.9-3.1\left(G^{\prime \prime}\right)$, and 7.6-11 $\left(G^{\prime}\right)$ and $0.5-4.2\left(G^{\prime \prime}\right)$ before freeze-drying and $6.1-12\left(G^{\prime}\right)$ and $2.3-3.3\left(G^{\prime \prime}\right)$, 3.3-6.5 $\left(G^{\prime}\right)$ and 0.4-2.4 $\left(G^{\prime \prime}\right)$, and 7.5-13 $\left(G^{\prime}\right)$ and 1.0-2.3 $\left(G^{\prime \prime}\right)$ after freeze-drying. (C) Mass percent and mole fraction of water over the concentration of trehalose and sucrose in FD NFC hydrogel formulation. Graphs represent mean \pm S.D., $n=3$. Abbreviations: NFC $=$ nanofibrillated cellulose, form. $=$ formulation, $\mathrm{FD}=$ freeze-dried/freeze-drying, $G^{\prime}=$ shear loss modulus, $G^{\prime \prime}=$ shear storage modulus, $\mathrm{mM}=$ millimolar.

$\mathrm{kJ} / \mathrm{mol}$ ). By taking the average value from the duplicate systems and calculating the relative difference between the means, sucrose had a $\sim 22 \%$ higher attraction to cellulose than trehalose in the hydrophobic cellulose plane system. The corresponding differences in the hydrophilic crystalline and the amorphous plane systems were $\sim 11 \%$ and $\sim 20 \%$, respectively. Analysis of the hydrogen bonds between NFC and biomolecules revealed that there was not a significant difference between the number of hydrogen bonds of the biomolecules. Importantly, however, in all systems sucrose formed consistently $\sim 7-8 \%$ fewer hydrogen bonds with water when compared to trehalose (SI, Table S3). This finding is also in accordance with the binding free energies of these sugars.

The penetration of water molecules into the NFC system was studied in the amorphous systems. Water molecules penetrated the amorphous cellulose plane readily, unlike the sugars despite their attraction to the cellulose-water interface. Without biomolecules, on average $263( \pm 7$ SD) water molecules resided inside a $2 \mathrm{~nm}$ slice of amorphous cellulose. Trehalose did not show any effect on the penetration $(263 \pm 5$ and $262 \pm 5)$, whereas the presence of sucrose increased the water penetration to an average of $277(280 \pm 13$ and $274 \pm 8)$, which corresponds to an increase of $\sim 5 \%$. These results are visualized with the attractions of the biomolecules in Figure 5B.

\section{DISCUSSION}

Different drying methods for NFC hydrogel have been applied to obtain aerogel with an intact nanoscale structure and 3D network. ${ }^{3,4}$ In addition, subsequent reconstitution of the FD TEMPO [(2,2,6,6-tetramethylpiperidin-1-yl)oxyl]-oxidized NFC (ANFC) hydrogel has been studied, where the surface charge density of the NFC fibrils has decreased the aggregation of the structure and this way has facilitated the freeze-drying 


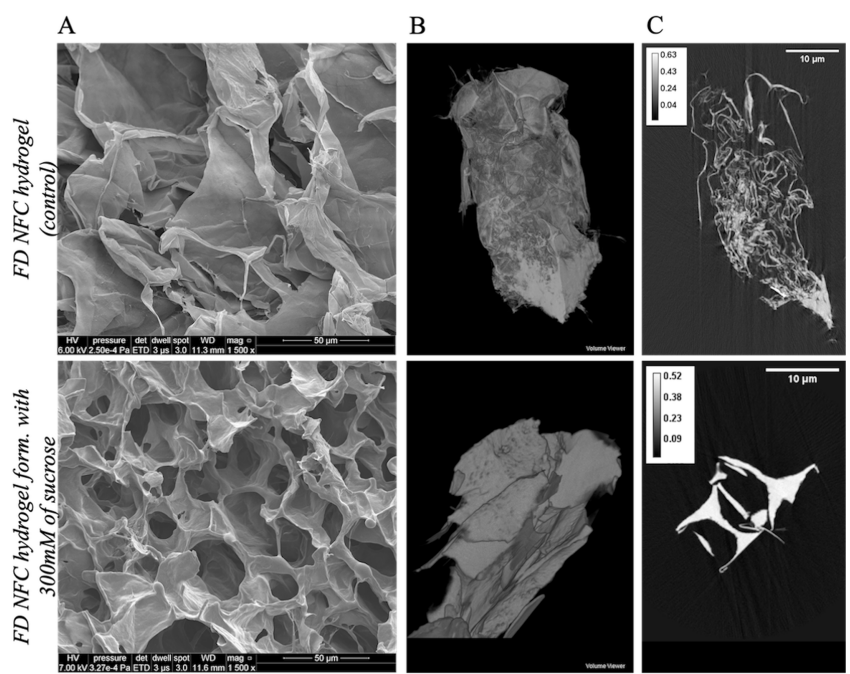

Figure 4. (A) SEM images of the FD NFC hydrogel (control, above) and the FD NFC hydrogel formulation with $300 \mathrm{mM}$ of sucrose (below). (B) Images of the 3D rendered volume of the FD samples (different parallel samples than the samples shown in panel A) shown by VolumeViewer of ImageJ obtained from PXCT data sets. (C) Slices through the tomograms of the FD samples shown in panel $\mathrm{B}$. The grayscale in the upper corner shows electron densities (units $\AA^{-3}$ ). The tomogram slice corresponding to the FD NFC hydrogel formulation with $300 \mathrm{mM}$ of sucrose showed a homogeneous electron density throughout the sample $\left(0.44-0.52 \AA^{-3}\right)$. Abbreviations: NFC = nanofibrillated cellulose, $\mathrm{FD}=$ freeze-dried, $\mathrm{mM}=$ millimolar.

process when compared to native one. ${ }^{13}$ However, with the comprehensive optimization of the freeze-drying process and by finding the optimal quantity of the biomolecules, we have shown now, for the first time, that the properties of the native NFC hydrogel after the freeze-drying and reconstitution can be preserved without further chemical modifications. Moreover, atomistic molecular dynamics simulations were utilized in studying the interactions between NFC and biomolecules (trehalose and sucrose) relevant in the present freeze-drying process. However, we must stress here that it is not possible to simulate the complex process of freeze-drying by employing atomistic or even superatom MD simulations at the moment since the size and time scales (the rate of temperature decrease or increase during the freeze-drying process in our case was $1 \mathrm{~K} /$ $\mathrm{min}$ ) associated with the process are currently out of reach of atomistic MD simulations. For example, simulating a large NFC system with the dimensions of hundreds of nanometers, decreasing content of water, morphological changes of NFC and the formation of water crystals taking place during the lengthy freeze-drying process in subzero temperatures is not possible with the current methodological and technological arsenal. In addition, such simulations are not feasible even with smaller system sizes (as reported in our article) because of the very low dynamics and diffusion of molecules when nearing the target freeze-drying temperatures reported here $(\sim 233 \mathrm{~K})$. In addition, the classical standard atomistic force fields (no electronic polarization) commonly used with lyoprotectants are usually parametrized in temperatures well above $273 \mathrm{~K}$, and it is therefore expected that non-natural behavior of lyoprotectants is taking place when these classical force fields are used in drastically different ways than their parametrization temperatures. Therefore, we examined a small NFC patch/fiber in water with different biomolecules mimicking the conditions of the systems before freeze-drying at room temperature, that is without a systematic removal of water or change in temperature. In this way we believe that we can still gather valuable information explaining some of the experimental results as shown and discussed in our article. Importantly, the simulation results highlighted that the sugars induced differences in the binding strength, hydrogen bonding, and the amount of water in the not FD system, which has not been studied before. Despite the simulations having not been carried out in conditions that resemble FD NFC, the results provide insight into the observed experimental data, which could be utilized in the designing of the formulations for freeze-drying in the future.

Although specific reasons for the lyoprotective properties of different excipients, especially for sugars, have not been truly clarified, different theories have been created to describe the behavior. $^{9-11}$ Commonly used lyoprotectant, trehalose, has been suggested to have the best capability in preserving the $3 \mathrm{D}$ structure of the macromolecules, such as proteins, during freezedrying due to its superior ability in displacing the hydrogen bonds between water and the sample. However, this statement did not reflect our results that showed sucrose to be more in line with this theory. Reconstituted FD NFC hydrogel formulation with $300 \mathrm{mM}$ of sucrose preserved the linear viscosity properties and similar viscoelastic features more efficiently than the one with trehalose. One reason for the better preservation properties of sucrose might be the higher attraction to nanocellulose fibers when compared with trehalose. This was evidenced by the MD simulations indicating higher free energy of binding for sucrose to the NFC surface in both crystalline systems that showed identical results in the bonding properties of the biomolecules. Hence, sucrose interacted more strongly with NFC forming a tighter, more uniform structure and perhaps this way had higher protectivity than trehalose. Higher interaction of sucrose was accompanied by a lower propensity of forming hydrogen bonds with water. We were not able to detect a difference between the hydrogen bonding of sucrose-NFC and trehalose-NFC through our simulations, suggesting that the increased attraction is caused by other interactions - or that the used simulation size was not sufficient. Regardless, as mentioned, sucrose had a lower number of hydrogen bonds to water than trehalose, which factors into the attraction results. The same type of phenomenon where sucrose was more attracted to the active component and displaced the water bonding was observed in the study of Prestrelski et al., ${ }^{36}$ in which residual water content was decreased in the sucrose-water solution after freeze-drying with an increasing amount of dry protein.

In addition to the water replacement hypothesis, trehalose is claimed to entrap water molecules more efficiently than other sugars and, in this way, it results in higher residual water content after the freeze-drying. ${ }^{11}$ However, FD NFC hydrogel formulations including $300 \mathrm{mM}$ or higher concentration of sucrose had a higher amount of residual water when compared with the ones including trehalose. Moreover, our results showed proportionality between increasing sucrose concentration and higher residual water content in the concentration range of 100 mM-300 mM, which was not the case with trehalose. These results agreed with the simulated water penetration of the amorphous NFC system, which was increased with the addition of sucrose. One explanation for the differences registered in the residual water contents might be, again, in the attraction preferences of these biomolecules: the higher the attraction of the biomolecule on the NFC surface was, the more water molecules were penetrated inside the NFC system. Since sucrose showed a higher attraction than trehalose to NFC, it 
A

NFC system with $300 \mathrm{mM}$ of trehalose

\section{NFC system with $300 \mathrm{mM}$ of sucrose}
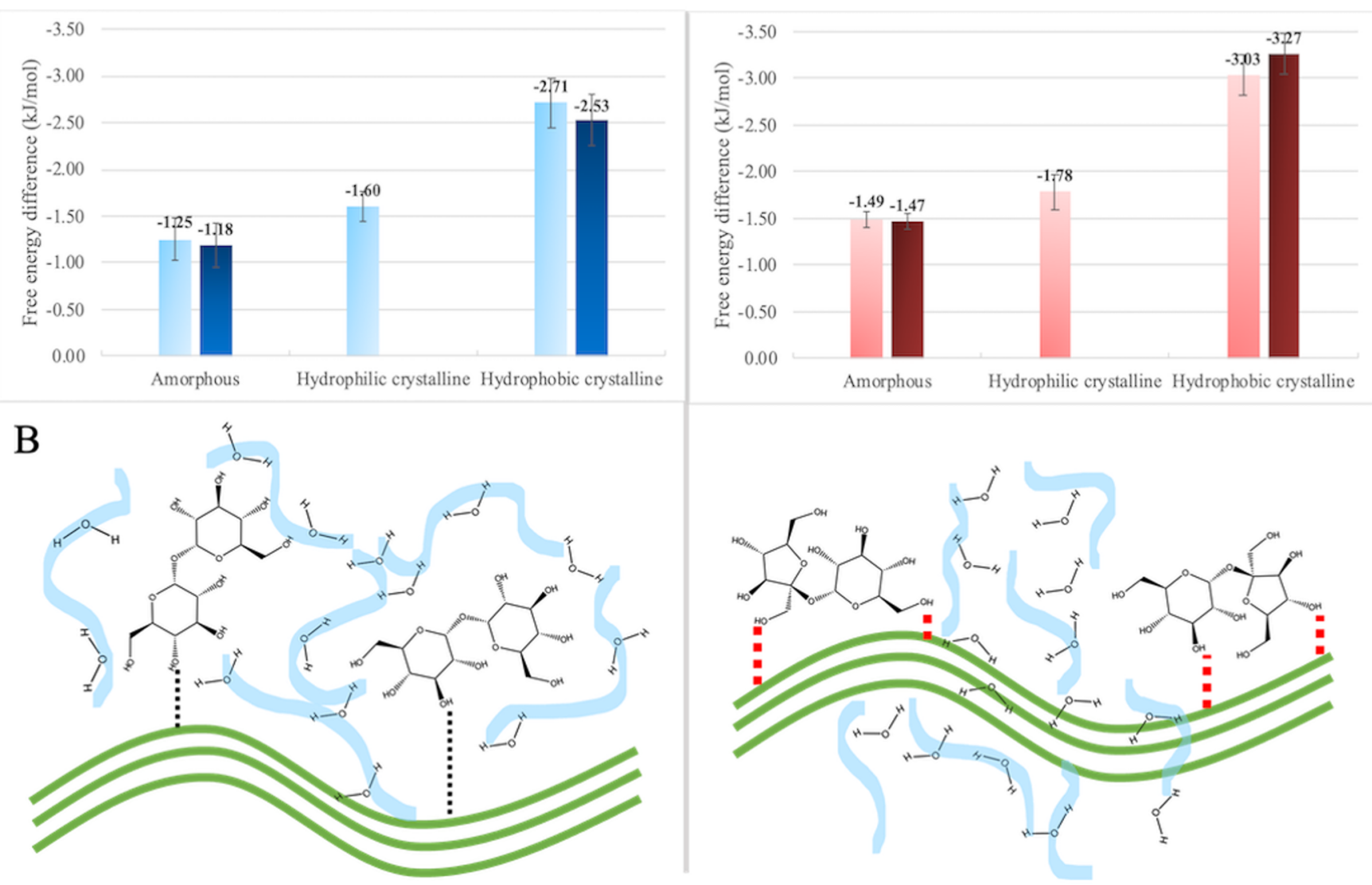

Figure 5. (A) Free energy difference between the NFC and biomolecules in all systems (amorphous and crystalline). Error bars are standard deviations produced from block averaging. Simulations in the amorphous system and hydrophobic system were implemented as duplicates (darker colored bars). (B) Schematic figure of the water penetration into the NFC system including $300 \mathrm{mM}$ of trehalose (left) or $300 \mathrm{mM}$ of sucrose (right). Change in the number of water molecules is compared to the pure water system with water molecules of $263( \pm 7)$. In the trehalose system, the number of penetrated water molecules was $262 \pm 5$ and $263 \pm 5$, whereas in the case of sucrose the number was $280 \pm 13$ and $274 \pm 8$. The number of water molecules was calculated within $1 \mathrm{~nm}$ of the $x y$-plane formed by the center of mass of cellulose in amorphous systems. The black arrows represent lower attraction between the sugars and cellulose chain and the red ones represent a strong attraction. Abbreviations: NFC = nanofibrillated cellulose.

captured water to a lesser extent, which was freely entered inside the NFC system while trehalose bonded more with water outside the system. This means that, although hydrogen bonds with water were formed in both systems, the location of them affected the final residual water content. The bound water with trehalose might be more feasibly desorbed during the freezedrying than with sucrose where the lower amount of water molecules was bonded with the sugar and more entered inside of the NFC system where stronger hydrogen bonds might have been formed. These observed differences between sucrose and trehalose could partly be explained by differences between the experimentally measured partition coefficient values $(\log P)$ of trehalose and sucrose noticed in the study of Mazzobre et al. ${ }^{37}$ $\log P$ value describes the lipophilicity of the substance, which is known to be low for trehalose and sucrose indicating relatively high water solubilities. $\log P$ value of sucrose was observed to be slightly higher $(-3.30 \pm 0.03)$ than with trehalose $(-3.77 \pm$ $0.05)$ at $20{ }^{\circ} \mathrm{C}$, suggesting that sucrose would have lower hydrophilicity and this way seek less proximity to water molecules and more with the amorphous hydrophobic sites of nanocellulose. This was in correlation with the biomoleculewater hydrogen bonding number abilities. To summarize, it might be that the addition of sucrose into the system enables stronger hydrogen bonding with water and this way increases the residual water content by interacting more strongly with the NFC than in the case of trehalose. The increasing concentration of sucrose was observed to be proportional with the increasing water content, which might saturate after a certain $\mathrm{NFC} /$ sugar ratio.

Both FD NFC hydrogel formulations formed organized porous structure evidenced by the SEM images, which could be explained by the formation of ice crystals during freeze-drying. ${ }^{4}$ Moreover, obtained PXCT images showed the FD NFC hydrogel formulation including sucrose to be homogeneous by having evenly formed $3 \mathrm{D}$ structure as well as the electron density in the narrow range throughout the whole sample. The homogeneous structure and organized porosity of the aerogel with the presence of sucrose might be valuable parameters to follow when considering the reconstitution back to the hydrogel. These results differed significantly from the FD control, having an unorganized structure and varying electron densities. Moreover, it is important to note that the differences between the observations of the parallel samples of the FD control refer to the low reproducibility and the changed properties of the sample after the freeze-drying without the presence of the suitable lyoprotectant. 
Based on this study, freeze-drying and reconstitution with preserved properties of native NFC hydrogel were most successful with the formulation including $300 \mathrm{mM}$ of sucrose as a lyoprotective biomolecule. Having sucrose as the only biomolecule with NFC hydrogel brings many benefits compared to other studied lyoprotectants; by eliminating the NFC hydrogel formulations having lower $T_{\mathrm{g}}{ }^{\prime}$ temperatures, primary temperature could be increased from -50 to $-42{ }^{\circ} \mathrm{C}$. This has a major impact from the time consumption and financial point of view since even $1{ }^{\circ} \mathrm{C}$ increase in the sample during freeze-drying could decrease the primary time up to $13 \% .{ }^{38}$ Besides, based on the MD simulations studies, the ability of water to penetrate more into amorphous NFC including sucrose than into the one with trehalose could be an advantage in the prevention of denaturation and the formation of the plasticizing effect into the FD system as well as in more feasible reconstitution of the FD NFC hydrogel. Based on the mass percent of the water in the FD sample with sucrose was however low enough to be stated as a dry product. Moreover, porous, organized morphology and physicochemical properties, such as iso-osmotic solution, $\mathrm{pH}$ neutral environment, and nontoxicity of NFC hydrogel formulation with sucrose could be utilized when considering further pharmaceutical applications for instance in freeze-drying of biologics and cells in the biomaterial in question.

\section{CONCLUSION}

For the first time, native NFC hydrogel was successfully FD and subsequently reconstituted with the presence of optimized biomolecule concentrations. NFC hydrogel formulation including $300 \mathrm{mM}$ of sucrose showed the most optimal preservation properties and enabled the formation of elegant, FD cake with a highly porous and organized structure with even electron densities throughout the FD sample. The experimental results were following the results produced by the atomistic molecular dynamics simulations and differences between the behavior of the biomolecules could be observed. Therefore, this study also demonstrated that a concerted use of MD simulations and experimental methods will enable a more feasible and comprehensive way to design the formulation and to predict possible outcomes in more complex freeze-drying applications of pharmaceuticals.

\section{ASSOCIATED CONTENT}

\section{SI Supporting Information}

The Supporting Information is available free of charge at https://pubs.acs.org/doi/10.1021/acsabm.1c00739.

More detailed information about the experimental section and results, Figures $\mathrm{S} 1-\mathrm{S} 7$, and Tables $\mathrm{S} 1-\mathrm{S} 3$ as presented in the text (PDF)

\section{AUTHOR INFORMATION}

\section{Corresponding Author}

Marjo Yliperttula - Division of Pharmaceutical Biosciences, Faculty of Pharmacy, University of Helsinki, 00014 Helsinki, Finland; (1) orcid.org/0000-0003-0726-5733;

Email: marjo.yliperttula@helsinki.fi

\section{Authors}

Elle Koivunotko - Division of Pharmaceutical Biosciences, Faculty of Pharmacy, University of Helsinki, 00014 Helsinki, Finland; 이이.org/0000-0002-0797-0187
Arto Merivaara - Division of Pharmaceutical Biosciences, Faculty of Pharmacy, University of Helsinki, 00014 Helsinki, Finland; (1) orcid.org/0000-0002-3917-3107

Akseli Niemelä - Division of Pharmaceutical Biosciences, Faculty of Pharmacy, University of Helsinki, 00014 Helsinki, Finland

Sami Valkonen - Division of Pharmaceutical Biosciences, Faculty of Pharmacy, University of Helsinki, 00014 Helsinki, Finland

Kalle Manninen - Division of Pharmaceutical Biosciences, Faculty of Pharmacy, University of Helsinki, 00014 Helsinki, Finland

Henrik Mäkinen - Department of Physics, University of Helsinki, 00014 Helsinki, Finland

Mira Viljanen - Department of Physics, University of Helsinki, 00014 Helsinki, Finland

Kirsi Svedström - Department of Physics, University of Helsinki, 00014 Helsinki, Finland

Ana Diaz - Paul Scherrer Institute, 5232 Villigen, Switzerland

Mirko Holler - Paul Scherrer Institute, 5232 Villigen, Switzerland

Jacopo Zini - Division of Pharmaceutical Biosciences, Faculty of Pharmacy, University of Helsinki, 00014 Helsinki, Finland

Lauri Paasonen - UPM Biomedicals, UPM-Kymmene Corporation, 00100 Helsinki, Finland

Ossi Korhonen - School of Phamacy, University of Eastern Finland, 70210 Kuopio, Finland

Simo Huotari - Department of Physics, University of Helsinki, 00014 Helsinki, Finland

Artturi Koivuniemi - Division of Pharmaceutical Biosciences, Faculty of Pharmacy, University of Helsinki, 00014 Helsinki, Finland

Complete contact information is available at:

https://pubs.acs.org/10.1021/acsabm.1c00739

\section{Author Contributions}

All authors have given approval to the final version of the manuscript. Author statement: Conceptualization by M.Y., A.K., E.K., A.M., and J.Z.; software by A.N., A.K., H.M., M.V., and S.H.; validation by all; investigation by E.K., A.M., S.V., and K.M.; resources by M.Y., S.H., and O.K.; writing original draft by E.K., A.M., A.N., A.K., A.D., and M.Y.; writing-review editing by all; supervision by M.Y. and A.K.; funding acquisition by M.Y. and S.H.

\section{Funding}

This study was financially supported by the UPM-Business Finland, GD-96 project, Finland for E.K., A.M., and A.N.; Emil Aaltonen Foundation No. 200141N Finland for A.M.; EVE ecosystem Dno 1842/31/2019 Business Finland for S.V.; Academy of Finland No. 314406 for J.Z.; NordicPOP to cover part of the materials and travel expenses for E.K., A.M., and O.K. The funders had no part in the designing of this research data analysis or arrangement of this manuscript.

\section{Notes}

The authors declare no competing financial interest.

\section{ACKNOWLEDGMENTS}

The Helsinki Electron microscopy unit at the Institute of Biotechnology (EMBI), University of Helsinki, is thanked for their support. We acknowledge the Paul Scherrer Institut, Villigen, Switzerland for the provision of synchrotron radiation beamtime at beamline cSAXS of the SLS. Authors acknowledge 
funding from NordForsk for the Nordic University Hub project \#85352 Nordic POP, Patient Oriented Products. A.M. acknowledges funding from Emil Aaltonen Foundation. Lisa Chinello and Giulia Scapin, University of Padova, Italy, funded by EU Erasmus exchange program, are thanked for their effort in helping with the experiments.

\section{ABBREVIATIONS}

NFC; nanofibrillated cellulose; FD; freeze-dried; MD; molecular dynamics; SEM; scanning electron microscopy; PXCT; ptychographic X-ray computed tomography.

\section{REFERENCES}

(1) Bhattacharya, M.; Malinen, M. M.; Lauren, P.; Lou, Y.-R.; Kuisma, S. W.; Kanninen, L.; Lille, M.; Corlu, A.; GuGuen-Guillouzo, C.; Ikkala, O.; Laukkanen, A.; Urtti, A.; Yliperttula, M. Nanofibrillar cellulose hydrogel promotes three-dimensional liver cell culture. J. Controlled Release 2012, 164, 291-298.

(2) Gaharwar, A. K.; Peppas, N. A.; Khademhosseini, A. Nanocomposite hydrogels for biomedical applications. Biotechnol. Bioeng. 2014, 111, 441-453.

(3) Jin, H.; Nishiyama, Y.; Wada, M.; Kuga, S. Nanofibrillar cellulose aerogels. Colloids Surf., A 2004, 240, 63-67.

(4) Peng, Y.; Gardner, D. J.; Han, Y. Drying cellulose nanofibrils: in search of a suitable method. Cellulose 2012, 19, 91-102.

(5) Fernandes Diniz, J. M. B.; Gil, M. H.; Castro, J. A. A. M. Hornification-its origin and interpretation in wood pulps. Wood Sci. Technol. 2004, 37, 489-494.

(6) Kawasaki, H.; Shimanouchi, T.; Kimura, Y. Recent development of optimization of lyophilization process. J. Chem. 2019, 2019, 1-14.

(7) Tang, M.; Wolkers, W. F.; Crowe, J. H.; Tablin, F. Freeze-dried rehydrated human blood platelets regulate intracellular $\mathrm{pH}$. Transfusion 2006, 46, 1029-1037.

(8) Patel, S. M.; Nail, S. L.; Pikal, M. J.; Geidobler, R.; Winter, G.; Hawe, A.; Davagnino, J.; Rambhatla Gupta, S. Lyophilized drug product cake appearance: what is acceptable? J. Pharm. Sci. 2017, 106, $1706-1721$.

(9) Oku, K.; Watanabe, H.; Kubota, M.; Fukuda, S.; Kurimoto, M.; Tsujisaka, Y.; Komori, M.; Inoue, Y.; Sakurai, M. NMR and Quantum Chemical Study on the $\mathrm{OH} \odot \odot \odot \pi$ and $\mathrm{CH} \odot \odot \odot \mathrm{O}$ Interactions between Trehalose and Unsaturated Fatty Acids: Implication for the Mechanism of Antioxidant Function of Trehalose. J. Am. Chem. Soc. 2003, 125, 12739-12748.

(10) Anhorn, M. G.; Mahler, H.; Langer, K. Freeze drying of human serum albumin (HSA) nanoparticles with different excipients. Int. J. Pharm. 2008, 363, 162-169.

(11) Lewis, L. M.; Johnson, R. E.; Oldroyd, M. E.; Ahmed, S. S.; Joseph, L.; Saracovan, I.; Sinha, S. Characterizing the freeze-drying behavior of model protein formulations. AAPS PharmSciTech 2010, 11, $1580-1590$.

(12) Arsiccio, A.; Paladini, A.; Pattarino, F.; Pisano, R. Designing the optimal formulation for biopharmaceuticals: a new approach combining molecular dynamics and experiments. J. Pharm. Sci. 2019, 108, 431-438.

(13) Paukkonen, H.; Kunnari, M.; Laurén, P.; Hakkarainen, T.; Auvinen, V.; Oksanen, T.; Koivuniemi, R.; Yliperttula, M.; Laaksonen, T. Nanofibrillar cellulose hydrogels and reconstructed hydrogels as matrices for controlled drug release. Int. J. Pharm. 2017, 532, 269-280.

(14) May, J. C.; Wheeler, R. M.; Etz, N.; Del Grosso, A. Measurement of final container residual moisture in freeze-dried biological products. Dev. Biol. Stand. 1992, 74, 153-164.

(15) Kauppinen, A.; Toiviainen, M.; Korhonen, O.; Aaltonen, J.; Järvinen, K.; Paaso, J.; Juuti, M.; Ketolainen, J. In-line multipoint nearinfrared spectroscopy for moisture content quantification during freezedrying. Anal. Chem. 2013, 85, 2377-2384.

(16) Auvinen, V.-V.; Merivaara, A.; Kiiskinen, J.; Paukkonen, H.; Laurén, P.; Hakkarainen, T.; Koivuniemi, R.; Sarkanen, R.; Ylikomi, T.;
Laaksonen, T.; Yliperttula, M. Effects of nanofibrillated cellulose hydrogels on adipose tissue extract and hepatocellular carcinoma cell spheroids in freeze-drying. Cryobiology 2019, 91, 137-145.

(17) Weng, L.; Ziaei, S.; Elliott, G. D. Effects of water on structure and dynamics of trehalose glasses at low water contents and its relationship to preservation outcomes. Sci. Rep. 2016, 6, 1-10.

(18) Pfeiffer, F. X-ray ptychography. Nat. Photonics 2018, 12, 9-17. (19) Holler, M.; Raabe, J.; Diaz, A.; Guizar-Sicairos, M.; Wepf, R.; Odstrcil, M.; Shaik, F. R.; Panneels, V.; Menzel, A.; Sarafimov, B.; Maag, S.; Wang, X.; Thominet, V.; Walther, H.; Lachat, T.; Vitins, M.; Bunk, O. OMNY - a tOMography nano crYo stage. Rev. Sci. Instrum. 2018, $89,043706$.

(20) Odstrčil, M.; Lebugle, M.; Guizar-Sicairos, M.; David, C.; Holler, M. Towards optimized illumination for high-resolution ptychography. Opt. Express 2019, 27, 14981-14997.

(21) Wakonig, K.; Stadler, H.; Odstrčil, M.; Tsai, E. H.; Diaz, A.; Holler, M.; Usov, I.; Raabe, J.; Menzel, A.; Guizar-Sicairos, M. PtychoShelves, a versatile high-level framework for high-performance analysis of ptychographic data. J. Appl. Crystallogr. 2020, 53, 574-586.

(22) Thibault, P.; Guizar-Sicairos, M. Maximum-likelihood refinement for coherent diffractive imaging. New J. Phys. 2012, 14, 063004.

(23) Holler, M.; Odstrcil, M.; Guizar-Sicairos, M.; Lebugle, M.; Muller, E.; Finizio, S.; Tinti, G.; David, C.; Zusman, J.; Unglaub, W.; Bunk, O.; Raabe, J.; Levi, A. F. J.; Aeppli, G. Three-dimensional imaging of integrated circuits with macro-to nanoscale zoom. Nat. Electron. 2019, 2, 464-470.

(24) Stockmar, M.; Cloetens, P.; Zanette, I.; Enders, B.; Dierolf, M.; Pfeiffer, F.; Thibault, P. Near-field ptychography: phase retrieval for inline holography using a structured illumination. Sci. Rep. 2013, 3, 16.

(25) Odstrčil, M.; Holler, M.; Raabe, J.; Guizar-Sicairos, M. Alignment methods for nanotomography with deep subpixel accuracy. Opt. Express 2019, 27, 36637-36652.

(26) Diaz, A.; Trtik, P.; Guizar-Sicairos, M.; Menzel, A.; Thibault, P.; Bunk, O. Quantitative x-ray phase nanotomography. Phys. Rev. B: Condens. Matter Mater. Phys. 2012, 85, 020104.

(27) Schindelin, J.; Arganda-Carreras, I.; Frise, E.; Kaynig, V.; Longair, M.; Pietzsch, T.; Preibisch, S.; Rueden, C.; Saalfeld, S.; Schmid, B.; Tinevez, J.-Y.; White, D. J.; Hartenstein, V.; Eliceiri, K.; Tomancak, P.; Cardona, A. Biological imaging software tools. Nat. Methods 2012, 9, 676-682.

(28) Bregado, J. L.; Secchi, A. R.; Tavares, F. W.; de Sousa Rodrigues, D.; Gambetta, R. Amorphous paracrystalline structures from native crystalline cellulose: A molecular dynamics protocol. Fluid Phase Equilib. 2019, 491, 56-76.

(29) Huang, J.; MacKerell, A. D., Jr CHARMM36 all-atom additive protein force field: Validation based on comparison to NMR data. J. Comput. Chem. 2013, 34, 2135-2145.

(30) Jorgensen, W. L.; Chandrasekhar, J.; Madura, J. D.; Impey, R. W.; Klein, M. L. Comparison of simple potential functions for simulating liquid water. J. Chem. Phys. 1983, 79, 926-935.

(31) Parrinello, M.; Rahman, A. Polymorphic transitions in single crystals: A new molecular dynamics method. J. Appl. Phys. 1981, 52, $7182-7190$

(32) Bussi, G.; Donadio, D.; Parrinello, M. Canonical sampling through velocity rescaling. J. Chem. Phys. 2007, 126, 014101.

(33) Darden, T.; York, D.; Pedersen, L. Particle mesh Ewald: An N. $\log (\mathrm{N})$ method for Ewald sums in large systems. J. Chem. Phys. 1993, 98, 10089-10092.

(34) Bekker, H.; Berendsen, H.; Dijkstra, E. J.; Achterop, S.; Van Drunen, R.; Van der Spoel, D.; Sijbers, A.; Keegstra, H.; Reitsma, B.; Renardus, M. Gromacs-a parallel computer for molecular dynamics simulations. Physics computing. World Scientific Singapore 1993, 92, $252-256$.

(35) Diaz, A.; Malkova, B.; Holler, M.; Guizar-Sicairos, M.; Lima, E.; Panneels, V.; Pigino, G.; Bittermann, A. G.; Wettstein, L.; Tomizaki, T.; Bunk, O.; Schertler, G.; Ishikawa, T.; Wepf, R.; Menzel, A. Threedimensional mass density mapping of cellular ultrastructure by 
ptychographic X-ray nanotomography. J. Struct. Biol. 2015, 192, 461469.

(36) Prestrelski, S. J.; Tedeschi, N.; Arakawa, T.; Carpenter, J. F. Dehydration-induced conformational transitions in proteins and their inhibition by stabilizers. Biophys. J. 1993, 65, 661-671.

(37) Mazzobre, M. F.; Román, M. V.; Mourelle, A. F.; Corti, H. R. Octanol-water partition coefficient of glucose, sucrose, and trehalose. Carbohydr. Res. 2005, 340, 1207-1211.

(38) Pikal, M. J. Freeze-drying of proteins. Part I: process design. BioPharm. 1990, 3, 18-27. 